\title{
Mediastinal tube placement in a premature infant with cardiorespiratory derangement due to ventilator associated pneumomediastinum
}

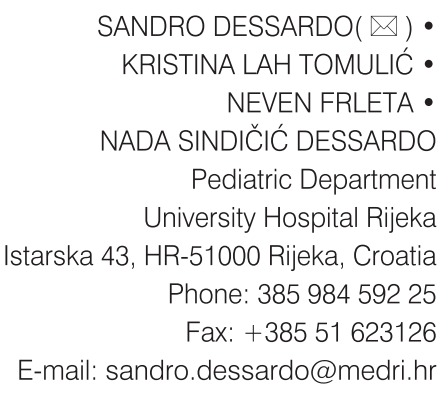

\section{SANDRO DESSARDO - KRISTINA LAH TOMULIĆ • NEVEN FRLETA • NADA SINDIČIĆ DESSARDO}

\begin{abstract}
While mediastinal free air in the ventilated newborn is usually benign, tension pneumomediastinum can lead to further cardiorespiratory compromise due to the compression of mediastinal structures, including the heart and large blood vessels. The authors present a case of life-threatening pneumomediastinum in a ventilated preterm leading to abrupt onset of cardiorespiratory failure. An 8 French $(\mathrm{Fr})$ drainage catheter was placed in the anterior mediastinum using the 2nd right intercostal space as an insertion site, with prompthemodynamic improvement. A brief description of the drainage technique and a literature review is presented.
\end{abstract}

Key words: hemodynamics, mechanical ventilation, pneumomediastinum, pneumothorax, thoracocentesis

\section{Introduction}

The first description of pneumomediastinum in the medical literature was related to a traumatic injury in 1819 . More than one hundred years later, in 1939, Hamman, for the first time, reported a spontaneous pneumomediastinum. Although a spontaneous pneumomediastinum is quite a rare entity in pediatric practice, in a NICU (Neonatal Intensive Care Unit) / PICU (Pediatric Intensive Care Unit) setting, pneumomediastinum can be observed more often, as a complication of mechanical ventilatory support. Pneumomediastinum rarely leads to clinically significant complications, and even in ventilated newborns it is a usually a benign entity. (1) Nevertheless, tension pneumomediastinum can severely compromise both respiratory and cardiac function. The elevated mediastinal pressure leads to diminished cardiac output because of compromised venous return. Isolated left ventricular inflow obstruction can be seen as a result of the increased juxtacardiac pressure in the pneumomediastinum. (2) The accepted explanation for the development of pneumomediastinum is that free air moves from ruptured alveoli along peribronchial vascular sheaths toward the lung hilum from where it extends within the mediastinum. From there, the free air can move in different directions eventually causing pneumothorax, pneumopericardium, pneumoperitoneum or subcutaneous emphysema which can additionally compromise the patient's cardiorespiratory status. Chest radiography is a mainstay for the diagnosis of pneumomediastinum, by revealing free air in the mediastinal space which outlines the normal anatomical structures and produces typical x-ray signs: a ring around the artery, thymic sail sign and continuous diaphragm sign. When free air in the mediastinum causes hemodynamic compromise, drainage is mandatory. The hemodynamic improvement resulting from drainage of the pneumomediastinum can be beneficial in patients who already have the propensity to develop multiple organ failure, since it can be exacerbated by the reduced cardiac output. (3) In hemodynamically unstable patients, pneumomediastinum as well as pneumothorax can further compromise cardiac and respiratory function. It is crucial to understand that after drainage, hemodynamic stability may be maintained without additional fluid administration or higher doses of vasopressors. (4)

\section{Case report}

Our patient was a preterm newborn of 
28 weeks gestational age and weighing 1060 grams at birth. He developed a typical clinical picture of respiratory distress syndrome so one dose of exogenous porcine surfactant was given and mechanical ventilation started. On day 10 a loud systolic murmur was heard, suggesting an opening of the ductus arteriosus, which was confirmed by echocardiography, and a PDA (Patent Ductus Arteriosus) closure protocol with indomethacin and frusemide was started. At this stage, the baby was under conventional ventilatory support, Pressure Support Ventilation with Volu-

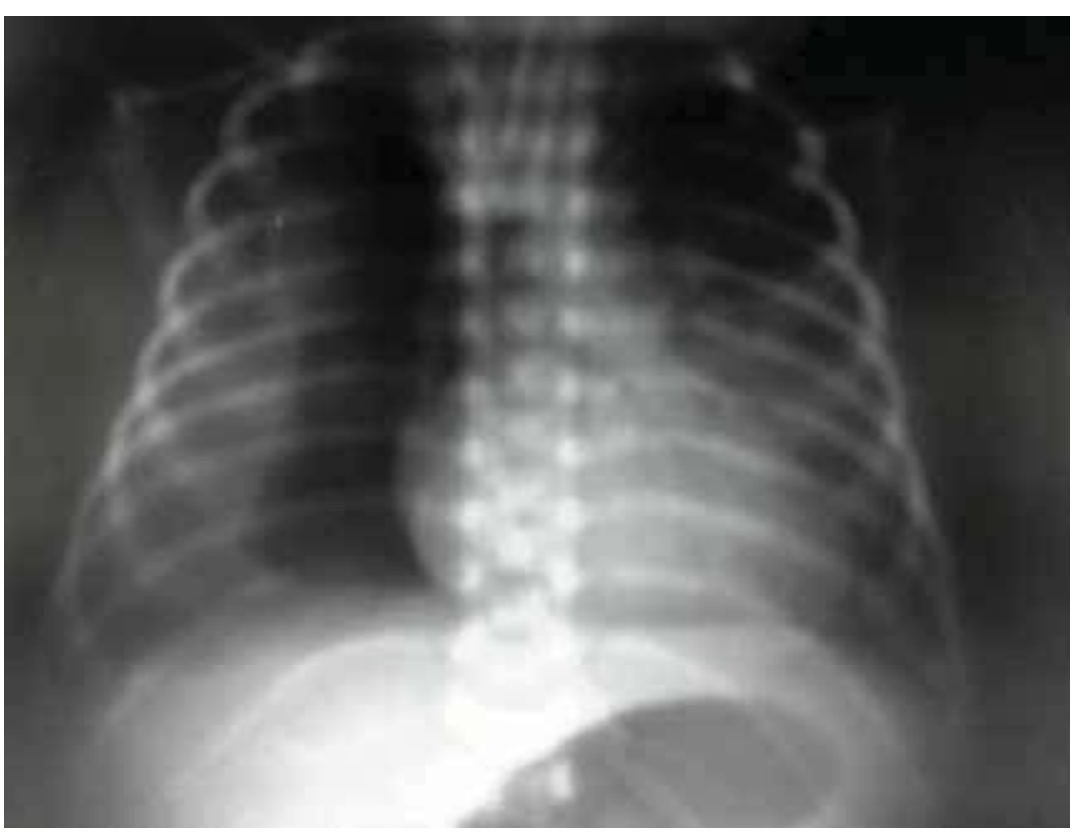

Figure 1. Thoracic x-ray prior to catheter insertion - a wide area of free mediastinal air, along the right cardiac border, evolving into a right tension pneumothorax.

me Guarantee option delivered by a Draeger babylog 8000 plus. The target Tidal Volume was $6 \mathrm{ml} / \mathrm{kg}$, PEEP (Positive End Expiratory Pressure) of $4 \mathrm{~cm}$ $\mathrm{H}_{2} \mathrm{O}$, PIP (Peak Inspiratory Pressure) of $18 \mathrm{~cm} \mathrm{H} \mathrm{H}_{2} \mathrm{O}$. Progressive deterioration of the baby's hemodynamic and respiratory status occurred. The pulse oximetry reading dropped from 95\% to $82 \%$, despite an increase in $\mathrm{FiO}_{2}$ (Fraction of Inspired Oxygen) from 0.35 to 0.8 . The systolic pressure values collected by a NIBP (Non Invasive
Blood Pressure) monitor decreased from around $55-60 \mathrm{~mm} \mathrm{Hg}$ to about $40 \mathrm{~mm} \mathrm{Hg}$. There was an increase in respiratory rate accompanied by visible intercostal retractions. There was no asymmetry of breath sounds. The endotracheal tube was patent with no signs of intraluminal obstruction. A thoracic $x$-ray was immediately performed, showing free air along the right cardiac border shifting the collapsed right lung toward the lateral thoracic wall (figure 1). A silastic 8 French (Fr) drainage catheter was placed in the upper anterior mediastinum using the ned from the ventilator, and two days later the mediastinal drainage tube was removed. The baby was transferred from the NICU to the neonatology ward, and dismissed from hospital at about 37 weeks of postconceptional age.

\section{Discussion}

The term „spontaneous pneumomediastinum"is reserved for cases of mediastinal free air that do not occur during or after chest trauma, thoracic surgery, endotracheobronchial procedures and mechanical ventilation. (5) Spontaneous pneumomediastinum is very seldom associated with severe cardiorespiratory compromise, if any. On the other hand tension pneumomediastinum, associated with mechanical ventilation, can severely compromise both respiratory and cardiac function and lead to acute cardiac failure. In our patient we observed a rapid deterioration of both respiratory and cardiac performance and we concluded that immediate mediastinal drainage was mandatory. Although different methods of mediastinal drainage such as tracheostomy, cervical mediastinostomy, surgical mediastinostomy, sternotomy and intermittent needle aspiration have been extensively described in the literature, $(6,7,8)$ we decided for percutaneous insertion of an $8 \mathrm{Fr}$ thoracocentesis catheter. Turlapati et al. reported on a successful subxyphoid approach in a series of 25 patients. They did not use any imaging technique for catheter guiding. Other papers report on fluoroscopic or ultrasound guided percutaneous insertion of percutaneous mediastinal drainage catheters. $(9,10)$ In our patient we decided for a bed-side non- guided percutaneous approach for two reasons. First, the baby's condition was rapidly deteriorating and there was no time to further postpone the procedure. Second, the thoracic $x$-ray showed a large free-air column along the right cardiac margin which, from our point of view, critically reduced the possibility of error during the catheter insertion procedure. Since the pneumomediastinum was already evolving into a right sided tension pneumothorax we used a "high" 
approach through the second intercostal space in order to favour the evacuation of air. We believe that, according to the already explained mechanism of free air moving inside the thorax, air should be evacuated from the site of maximal accumulation, irrespectively of the initial origin, and it should be done immediately if the patient's condition deteriorates rapidly, especially if there is a rapid decrease in cardiac output.

Based on the reviewed literature and on our experience we conclude that decompression of the mediastinum can be safely and rapidly achieved using a bed-side percutaneous approach, with or without additional imaging assistance. Free air under tension in the thoracic cavity should be removed immediately and "field" techniques are safe and effective in the NICU/PICU setting, equally or even more so than in the emergency medicine environment.

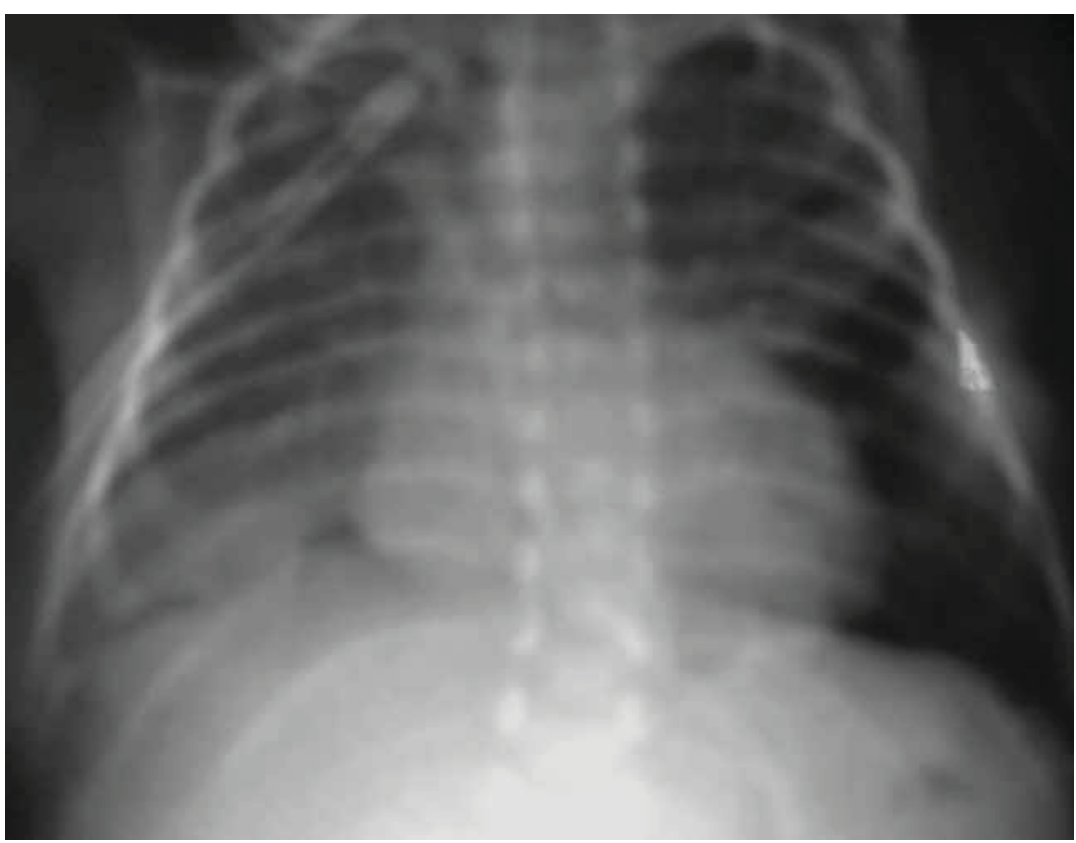

Figure 2. Thoracic x-ray after catheter insertion - complete re-expansion of the right lung.

\section{REFERENCES}

1. Hauri-Hohl A, Baenzigar O, Frey B. Pneumomediastinum in the neonatal and pediatric intensive care unit. Eur J Pediatr 2008;167:415-8.

2. Case CL, Oslizlok P, Fyfe D, Gilette PC. Neonatal pneumomediastinum with isolated mitral obstruction. Arch Dis Child 1990;65:56-8.

3. Livingston DH. Management of the surgical patient in MOSF. Am J Surg 1993;165(2A Suppl):8-13.

4. Turlapati KM, Spear RM, Peterson BM. Mediastinal tube placement in children with pneumomediastinum: Hemodynamic changes and description of technique. Ped Crit Care 1996;24(7):1257-60.

5. Chen CJ, Hsu ML, Diau GY, Fan HC, L SH, Lin WJ, et al. Neonatal spontaneous pneumomediastinum. J Med Sci 2003;23(1):49-52.

6. Beg MH, Reyazzudin MM. Traumatic tension pneumomediastinum mimicking cardiac tamponade. Thorax 1988;43:576-7.

7. Unger DL, Pifarre R. Tracheotomy for subcutaneous emphysema and pneumomediastinum complicating asthma. Chest 1972;61:691-2.

8. Thiebault DW, Lachman RS, Laul VR. Pulmonary interstitial emphysema, pneumomediastinum and pneumothorax. Am J Dis Chil 1973;126:611-4.

9. Chau HH, Kwok PC, Lai AK, Fan TW, Chan SC, Miu TY, et al. Percutaneous relief of tension pneumomediastinum in a child. Cardiovasc Intervent Radiol 2003;26(6):561-3.

10. Mohamed IS, Lee YH, Yamout SZ, Fakir S, Reynolds AM. Ultrasound guided percutaneous relief of tension pneumomediastinum in a 1-day old newborn. Arch Dis Child Fetal Neonatal Ed 2007;92(6):458. 